\title{
Understanding magnetism and electromagnetism and their implicaions: A tribute to David W. Strangway
}

\author{
Guest Editors \\ Jafar Arkani-Hamed \\ University of Toronto, Toronto, Ontario, Canada \\ David J. Dunlop \\ University of Toronto, Toronto, Ontario, Canada \\ John W. Geissman \\ University of Texas at Dallas, Richardson, Texas, USA \\ Özden Özdemir \\ University of Toronto, Toronto, Ontario, Canada \\ David T.A. Symons \\ University of Windsor, Windsor, Ontario, Canada
}

